\title{
Energy Efficient MAC Scheme for Wireless Sensor Networks with High-Dimensional Data Aggregate
}

\author{
Seokhoon Kim, ${ }^{1}$ Hangki Joh, ${ }^{2}$ Seungjun Choi, ${ }^{3}$ and Intae Ryoo ${ }^{2}$ \\ ${ }^{1}$ Department of Mobile Communications Engineering, Changshin University, 262 Paryong-ro, Masanhoiwon-gu, Changwon-si, \\ Gyeongsangnam-do 630-764, Republic of Korea \\ ${ }^{2}$ Department of Computer Engineering, Kyung Hee University, 1732 Deogyeong-daero, Giheung-gu, Yongin-si, \\ Gyeonggi-do 446-701, Republic of Korea \\ ${ }^{3}$ The Aerospace Strategy \& Technology Institute, Yonsei University, 50 Yonsei-ro, Seodaemun-gu, Seoul 120-749, Republic of Korea \\ Correspondence should be addressed to Intae Ryoo; itryoo@khu.ac.kr
}

Received 27 November 2014; Accepted 29 January 2015

Academic Editor: Sanghyuk Lee

Copyright (C) 2015 Seokhoon Kim et al. This is an open access article distributed under the Creative Commons Attribution License, which permits unrestricted use, distribution, and reproduction in any medium, provided the original work is properly cited.

\begin{abstract}
This paper presents a novel and sustainable medium access control (MAC) scheme for wireless sensor network (WSN) systems that process high-dimensional aggregated data. Based on a preamble signal and buffer threshold analysis, it maximizes the energy efficiency of the wireless sensor devices which have limited energy resources. The proposed group management MAC (GMMAC) approach not only sets the buffer threshold value of a sensor device to be reciprocal to the preamble signal but also sets a transmittable group value to each sensor device by using the preamble signal of the sink node. The primary difference between the previous and the proposed approach is that existing state-of-the-art schemes use duty cycle and sleep mode to save energy consumption of individual sensor devices, whereas the proposed scheme employs the group management MAC scheme for sensor devices to maximize the overall energy efficiency of the whole WSN systems by minimizing the energy consumption of sensor devices located near the sink node. Performance evaluations show that the proposed scheme outperforms the previous schemes in terms of active time of sensor devices, transmission delay, control overhead, and energy consumption. Therefore, the proposed scheme is suitable for sensor devices in a variety of wireless sensor networking environments with high-dimensional data aggregate.
\end{abstract}

\section{Introduction}

The wireless sensor device is one of the core components of ubiquitous sensor network (USN) systems. Sensors connected by wireless communication technologies act as intermediary between people and things in ubiquitous computing environments. The ultimate goal of WSN is to gather interesting information on environment factors or object conditions on behalf of human being because sensor devices may be deployed in inaccessible terrains and even in hazardous places. To achieve this goal, wireless sensor devices have been designed and characterized as follows. Firstly, they should be realized at low cost. Secondly, they should have self-organizational capability [1]. Thirdly, they should be implemented as small as possible. Lastly, but most importantly, they should have long lifetime. This last feature of sensor device is the key challenge for the successful deployment of WSN systems as the sensor devices are equipped with limited energy resource and it is impractical to replace or recharge their batteries. Therefore, unlike the existing wireless communications devices that focus on transmission efficiency, the energy efficiency of sensor devices should be prioritized in WSNs. That is, energy efficient wireless sensor devices play a very important role in realizing robust and long-lasting WSNs. In addition, in order to ensure that the WSN does its role successfully, it is critical to maintain the distribution of active (or operating) sensor devices evenly in the whole target field rather than just keeping the number of active sensor devices as many as possible.

Wireless sensor devices consist of physical and logical layers to communicate with other sensor devices or with sink node in the corresponding WSN. Among these layers, 
medium access control (MAC) layer is closely related with energy efficiency of the sensor devices. MAC layer guarantees successful communications among wireless sensor devices by using MAC protocol for performing error and flow controls as well as for managing communication resources, which results in major energy consumption of sensor devices [2]. In other words, MAC protocol determines the lifetime of WSNs. Existing legacy MAC protocols have been designed to guarantee some degree of quality of services (QoS) such as maximum data throughput, minimum transmission delays, and fairness among the devices. In WSNs, however, MAC protocol should be designed in a different way due to the energy resource limitations.

To date, researchers have proposed numerous MACs. For example, MAC protocols that use multiple channels such as those in $[3,4]$ show good performances when there are few or no multichannel system collisions. But they suffer from their intrinsic complex wireless communication mechanisms, which waste energy. Therefore, MAC protocol design for WSNs is changed to use a single and robust channel [5]. Time division multiple access (TDMA) based MAC protocols [6-8] perform slot reservation and scheduling and hence no collisions, which naturally reduces energy expenditure. TDMA based MAC protocols, however, have intrinsic limitations which include the following: (i) the need for global synchronization, (ii) the need for knowing the schedule of neighboring devices, (iii) transmitting and receiving schedule which is continuously changing depending on topological database and traffic conditions, and (iv) changes in network topology as well as in number of sensor devices which cause very large control message overheads [9-11].

Contention-based MAC protocols have also been proposed to support energy efficiency of sensor nodes. The approach in [12] proposes a scheme to turn the wireless communication module on and off periodically by using preamble sensing of low level carrier to minimize energy consumption of the device. Also, the approach in [13] reduces energy expenditure by adaptively changing the preamble length depending on network traffics. Unfortunately, they have been designed for sporadic traffic condition and for the case that sending device knows the sampling schedule of receiving device, respectively. S-MAC [14] which has been designed especially for WSN is one of the contentionbased MAC protocols and uses time slot concept to reduce energy consumption. T-MAC [15] uses adaptable duty cycle to improve energy efficiency, whereas S-MAC uses fixed duty cycle. Furthermore, E2-MAC [16] improves the energy efficiency of T-MAC by regulating the data transmission of sensor nodes based on buffer threshold concept. Although these MAC protocols have been well designed for sensor nodes, they still do not guarantee the most important condition of successful deployment of WSNs, the long lifetime. Existing protocols focus on minimizing sensor node energy consumption, not on maximizing the lifetime of the whole WSN.

To this end, we present group management MAC (GMMAC), a novel, energy efficient, and sustainable medium access control technique for use in most WSNs. In GMMAC, sensor devices are divided into different groups depending on the separation distance from the sink node of the corresponding WSN. Sensor groups are assigned different buffer thresholds and different preamble signals to send data to devices in other groups and eventually to the sink node. For urgent data delivery, buffer threshold rule is not applied to data traffics with a high priority. In addition, GM-MAC designs improved control signals and timers to overcome the problem of both idle and unnecessary listening. By doing this, GM-MAC minimizes the control message overheads, data transmission delays, and the average energy consumption of the entire sensor devices in the target field, which results in the long lifetime of the corresponding WSN. The rest of this paper is structured as follows. Section 2 discusses related works. Section 3 describes our proposed scheme, and Section 4 shows the performance evaluation of the proposed GM-MAC scheme. Finally, we offer concluding remarks in Section 5.

\section{Related Works}

Sensor-MAC (S-MAC) is a foundational and important MAC protocol which is explicitly designed for WSNs. Generally, in WSNs, nodes are in idle state most of the time. And the idle listening operation of sensor nodes results in the main reason of a waste of node energy. To solve this problem, SMAC decreases idle listening time by making sensor node enter into sleep mode periodically based on duty cycle. SMAC also tries to reduce the waste of energy caused by collision, overhearing, and control packet overhead [17]. But there exists a tradeoff between data throughput and delay performance in S-MAC [18]. Timeout MAC (T-MAC) [15], an enhanced version of S-MAC, introduces an adaptive duty cycle by dynamically ending the active part of it in order to handle load variations in time and location. In this way, TMAC reduces the amount of energy wasted on idle listening, in which sensor nodes wait for potentially incoming messages while still maintaining a reasonable throughput. Compared to S-MAC, T-MAC shows similar results under homogeneous traffic condition and outperforms S-MAC in a scenario with variable traffic loads. T-MAC has an issue of early sleeping problem, which may cause data transmission delay. To solve this issue, T-MAC incorporates the schemes of future request-to-send (FRTS) and full-buffer priority. Although the early sleeping problem can be addressed with these solutions, delay shortening is achieved within one hop coverage. Data gathering MAC (D-MAC) considers a duty cycle for multiple hop chain environments to transmit the data continuously. In $\mathrm{D}-\mathrm{MAC}$, there is no request-to-send (RTS) or clear-to-send (CTS) procedures because the schedule is preconfigured. But it uses acknowledgment (ACK) frame for reliable delivery of data packets [19]. D-MAC, however, is applied to a special networking environment in which data are collected to the sink node. That is, it cannot support WSN with mobile sensor nodes. To further reduce the energy consumption of sensor node, energy efficiency-MAC (E2-MAC) specifies a threshold value to buffers in sensor nodes [16]. In this scheme, data can be transmitted to other nodes only if the buffer occupation exceeds a predetermined threshold value. Otherwise, noactivation-event timer for entering the sleep mode is set to a smaller value than that used in T-MAC. As E2-MAC falls into 
the sleep mode when a sensor node does not transmit RTS or data during the no-activation-event time, it can improve the energy efficiency of existing protocols. All the above MAC schemes, however, have focused only on minimizing energy consumption of a sensor node itself. For the successful deployment of WSNs, we have to maximize the lifetime of the whole WSN. In Section 3, we present a novel and sustainable GM-MAC scheme in detail.

\section{GM-MAC}

Wireless sensor nodes which have limited energy resources should survive as long as possible under various sensornetwork traffic conditions and topologies. With existing MAC schemes, the nearer sensor devices are located to the sink node the more they consume energy compared to the sensor devices that are further away from the sink node. In order to maximize the lifetime of the whole WSN, GM-MAC is explicitly designed to employ variable length preambles and buffer thresholds based on node-group management concept.

3.1. Preamble, Node Group, and Buffer Threshold Setup. GMMAC sets up the preamble and buffer threshold values of sensor devices just after they are randomly deployed in WSN. Obviously, the nodes that are closely located to the sink node receive the preamble first. These nodes are the sensor devices that are 1 hop away from the sink node. In other words, if a sensor device receives a preamble the size of which is the same as that of the sink node, it is the neighboring node of the sink node. These neighboring nodes are defined as node group 1, $G(1)$. Once a sensor device receives a preamble for the first time, it configures its preamble by constructing a new preamble and sends the new preamble to its neighboring nodes. In GM-MAC, constructing a new preamble means extending the synchronization part [20] of the currently received preamble. More specifically, the synchronization part of a newly created preamble is obtained by appending predetermined code repetitions to the synchronization part of the currently received preamble. This procedure continues until all the sensor devices in the target field configure their initial preambles as follows:

$$
\mathrm{PS}_{G(i)}= \begin{cases}\mathrm{PS}_{\text {sink_node }}+k_{1}, & \text { for } i=1, \\ \mathrm{PS}_{G(i-1)}+k_{1}, & \text { otherwise, }\end{cases}
$$

where $\mathrm{PS}_{G(i)}$ denotes the size of the preamble synchronization part used by the nodes in group $G(i)(i=1,2, \ldots, n)$, $\mathrm{PS}_{\text {sink_node }}$ is the size of the preamble synchronization part used by the sink node, and $k_{1}$ is the size of the predetermined code repetitions of the preamble synchronization part.

It might be possible to include hop count information or node's location information in data frame header rather than using variable length preambles. The proposed GMMAC scheme is, however, for wireless sensor networks in which sensor nodes are randomly moving, and locating all the sensor nodes in the target filed adds more complexities to the whole WSN design. Readers might be concerned about the energy consumption issue by introducing additional $k_{1}$ code repetitions to the base preamble of the sink node. But the

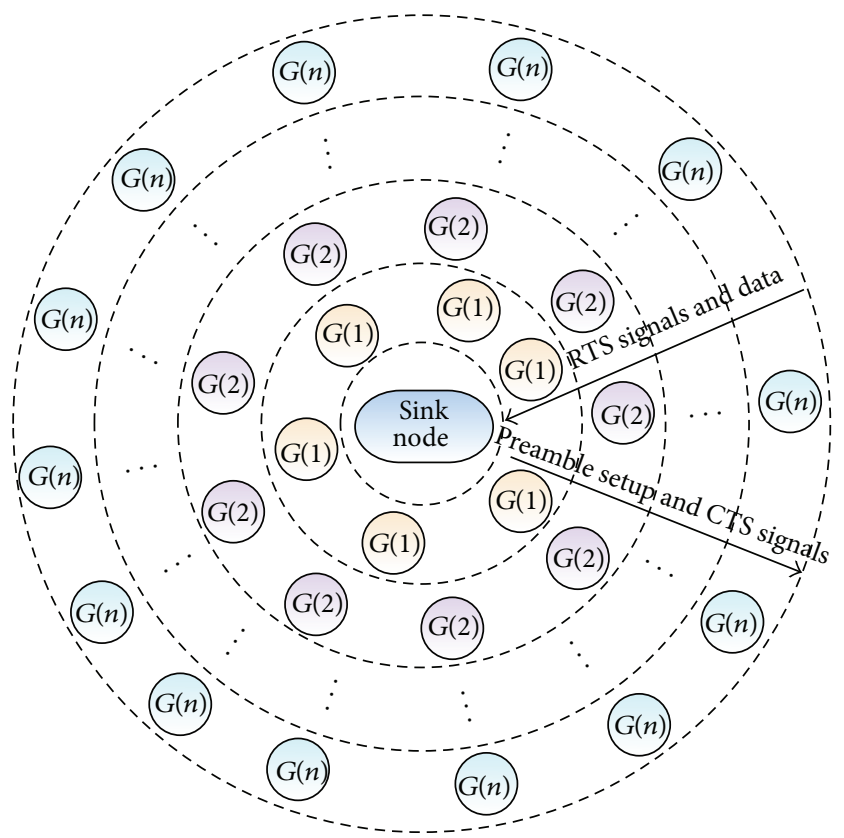

FIGURE 1: A sample WSN with $n$ node groups. Data transmission is done in the direction of the sink node.

overhead of $k_{1}$ code repetitions is negligible and acceptable if we consider the merits of using variable length preambles: figuring out nodes' location and maximizing the lifetime of the whole WSN. Actually, $k_{1}$ code repetitions are only 2 or 4 repetitions after 32 repetitions when using medium preamble type. More details about preamble design can be found in $[12,13,20]$, and the method for determining specific values of $\mathrm{PS}_{\text {sink_node }}$ and $k_{1}$ is left to the application developer.

Based on this preamble setup procedure, sensor devices in different node groups have different preamble sizes, which makes it possible to discern the node group from which the current data originates. Based on this preamble size setup procedure, data transmission is realized only in the direction of the sink node. If a sensor device verifies that the current data come from the same node group or upstream node group, it goes into sleep mode after short no-activationevent time. This feature can considerably reduce the amount of energy wasted on unnecessary listening as well as idle listening. Figure 1 shows a sample WSN with $n$ node groups. As illustrated in Figure 1, in GM-MAC, preamble setup and CTS signals are delivered from node group $G(i)$ to node group $G(i+1)$, and RTS signals and data are transmitted from node group $G(i+1)$ to node group $G(i)$.

At the time of configuring initial preamble size, sensor device also sets its buffer threshold values to be

$$
B_{G(i)}=k_{2} \times B_{t} \times \frac{(n+1-i)}{n},
$$

where $B_{G(i)}$ denotes the buffer threshold of the nodes in node group $G(i), k_{2}$ is the threshold weighting factor $(0<$ $\left.k_{2} \leq 1\right), B_{t}$ is the total buffer size of the sensor device, and $n$ is the total number of node groups in the target field. The threshold weighting factor $k_{2}$ has been introduced in 


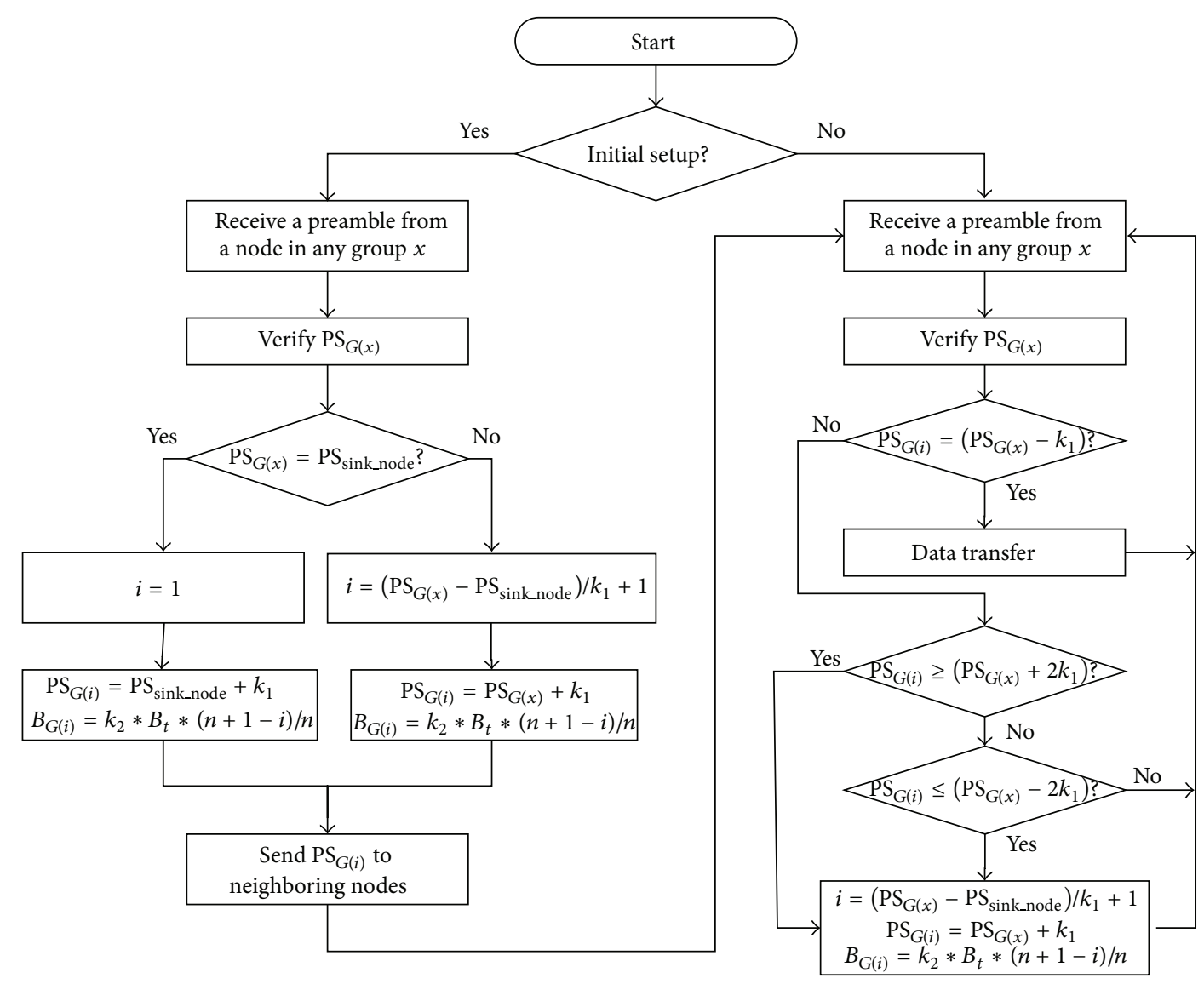

FIGURE 2: Preamble size, node group, and buffer threshold value setup and recalculation procedure by a node in node group $G(i)$. Initial setup is done when a sensor device is placed in the target field at the first time. Recalculation is done when a sensor device moves from node group $G(i)$ to any other node group $G(j)$, where $j \neq i$.

this paper as an implementation guide to handle various application scenarios under the condition that sensor devices have a certain factory-default buffer size $B_{t}$. It has to be specifically configured as a constant at the deployment stage of WSN depending on network size of the corresponding WSN and total buffer size of sensor nodes. Buffer threshold values of a sensor node are automatically adjusted by figuring out the node group $G(i)$ of that sensor node rather than by changing $k_{2}$. The method for determining optimum values of $k_{2}$ and $n$ is also left to the application developer.

As shown in (2), buffer threshold value of the nodes in each node group is configured to be in inverse proportion to the distance from the sink node. That is, sensor devices that are further away from the sink node have more chance to send their data to neighboring nodes than the nodes that are located nearer to the sink node. If the accumulated data that are collected by a sensor device itself and received from other nodes are equal to or exceed the buffer threshold, they are to be transmitted to neighboring nodes. Otherwise, the sensor device may receive data from the nodes in downstream node group or switch to sleep mode after a short timer value. In this way, GM-MAC maximizes the energy efficiency of the whole WSN while reducing the energy consumption of the individual sensor devices.
Preamble and buffer threshold do not have to change after the predescribed initial setup phase unless sensor devices move in the target field. Depending on the type of WSN applications, however, preamble size and buffer threshold values should be reconfigured. Furthermore, depending on the WSN situation, it may be necessary to add new sensor devices or substitute existing sensor devices in the target field. In order to support the scalability and self-organizational capability, GM-MAC performs preamble, node group, and buffer threshold recalculation procedure when necessary after the initial setup phase, as illustrated in Figure 2.

3.2. Improved Data Transmission Mechanism. GM-MAC uses improved control signals for reducing transmission delays and control overheads of the existing MAC schemes. The improved control signals include reservation RTS (R_RTS), booking CTS (B_CTS), and urgent data flag $D_{p}$.

R_RTS packet is sent from a sensor node in $G(i+1)$ to neighboring nodes in $G(i)$ when the node in $G(i+1)$ has the accumulated data that are equal to or exceed its buffer threshold $B_{G(i+1)}$ but fails to use the medium. With this R_RTS, the node in $G(i+1)$ has an opportunity to transmit its data to neighboring nodes in $G(i)$ for the next contention period. B_CTS packet is sent from a sensor node in $G(i)$ to 


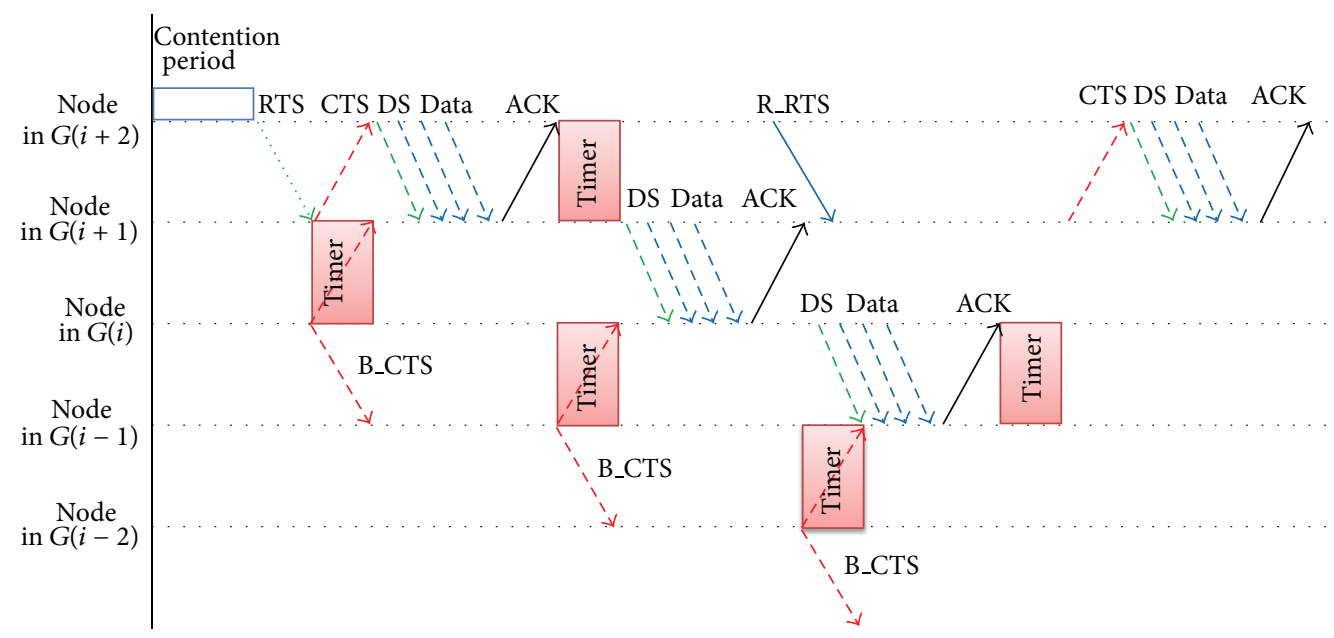

FIGURE 3: Data transmission using improved control signals, R_RTS and B_CTS. By notifying the neighboring nodes that a sensor device has data to send or is ready to receive data, energy management in the whole WSN is efficiently achieved.
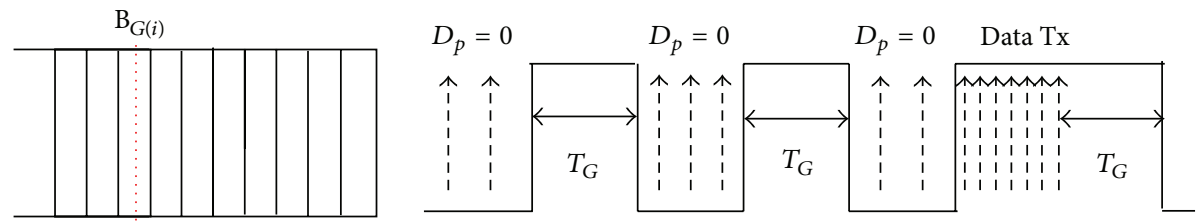

(a) Normal data transmission
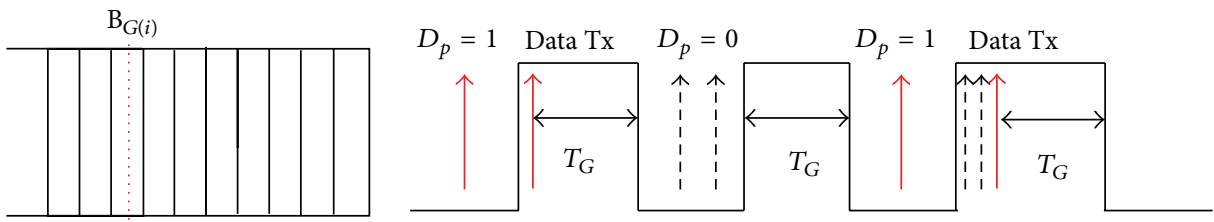

(b) Urgent data transmission

FIgURE 4: Normal and urgent data transmissions in GM-MAC. If a sensor device has urgent data with $D_{p}=1$, it sends data in its buffer regardless of the current buffer threshold value. $T_{G}$ is no-activation-event time.

neighboring nodes in $G(i+1)$ when the node in $G(i)$ has the accumulated data less than its buffer threshold $B_{G(i)}$. With his B_CTS, the node in $G(i)$ notifies neighboring nodes in $G(i+1)$ that it is ready for receiving data from downstream nodes rather than transmitting its data to upstream nodes. To prevent any other node from taking the medium during the next contention period, the node that sent the R_RTS or received the B_CTS transmits a small data-send (DS) packet just before the data transmission as in T-MAC. Data transmission with these improved control signals is illustrated in Figure 3.

The urgent data flag $D_{p}$ is used to indicate that a sensor device collects or senses urgent data. When a sensor device has urgent data, it should send its data immediately regardless of its current buffer occupation ratio. Figure 4 shows examples of normal and urgent data transmissions in case that the node has buffer threshold value of 7 .

Figure 5 is the flow chart which shows the whole data transmission procedure of the proposed GM-MAC scheme in active mode. As illustrated in Figure 5, only when the sensor device has ever received R_RTS, has data with $D_{p}=1$, or has accumulated data that exceed its $B_{G(i)}$, it can send its data to any upstream node. Otherwise, it goes into sleep mode after no-activation-event (timeout) time $T_{G}$.

\section{Performance Evaluation and Analysis}

To examine the performance of the proposed GM-MAC, energy efficiency comparisons with S-MAC, T-MAC, and E2$\mathrm{MAC}$ are carried out as for the indices such as active time of sensor devices, data transmission delays during active mode, control signal overheads, and energy consumption of sensor devices in each node group. In our performance evaluation model, we randomly distribute 1800 sensor nodes in a $1000 \times$ $1000 \mathrm{~m}^{2}$ field.

Table 1 shows reference parameters and variables used by the MAC schemes discussed in this paper. Table 2 shows power consumption values of radio chipset in a sensor node. 


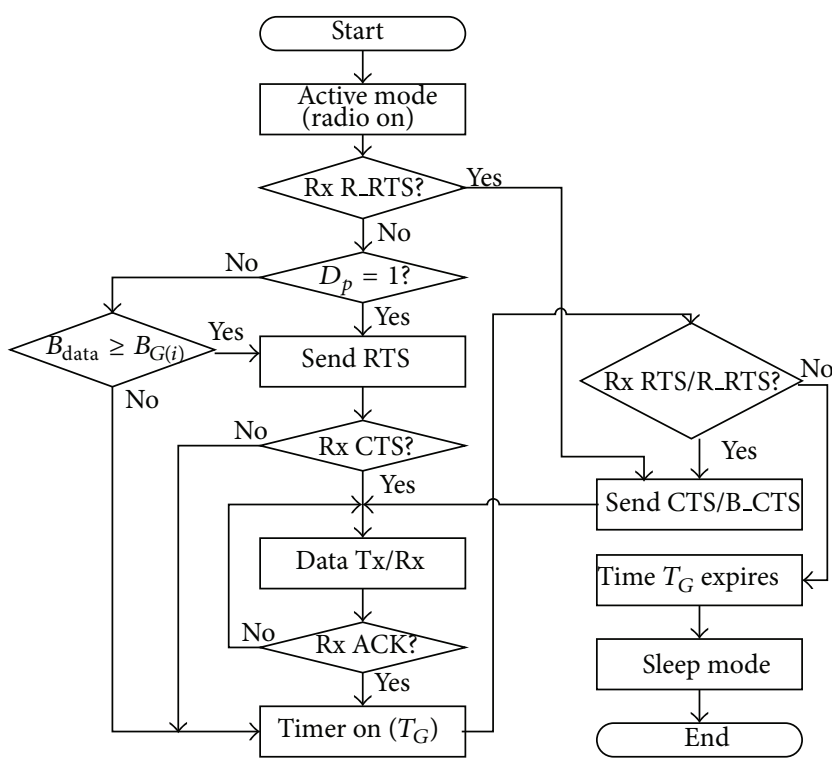

FIGURE 5: Flow chart of data transmission procedure of GM-MAC. $B_{\text {data }}$ is the amount of accumulated data in the buffer of this sensor device.

TABLE 1: Reference parameters and variables.

\begin{tabular}{lc}
\hline Parameter and variable & Value \\
\hline Contention slots & 256 \\
Duty cycle & $20 \%$ \\
Frame length & $1 \mathrm{~ms}$ \\
Probability variable & $0 \leq P(\rho) \leq 1,0 \leq P(\tau) \leq 1$ \\
Data distribution & Poisson, constant bit rate $(\mathrm{CBR})$ \\
Threshold variable & $0<k_{2} \leq 1$ \\
\hline
\end{tabular}

TABLE 2: Power consumption of radio chipset (tiny OS).

\begin{tabular}{lcc}
\hline Parameter & Meaning & Value \\
\hline$E_{s}$ & Power consumption for data transmission & $52.2 \mathrm{~mW}$ \\
$E_{r}$ & Power consumption for data reception & $56.4 \mathrm{~mW}$ \\
$E_{\text {id }}$ & Power consumption for idle listening & $56.4 \mathrm{~mW}$ \\
$E_{\text {sleep }}$ & Power consumption for sleep mode & 0 \\
\hline
\end{tabular}

As with any WSN MAC scheme, we assume that sensor node uses a CC2420 radio transceiver (operating in the $2.4 \mathrm{GHz}$ band), from Texas Instruments. According to its data sheet [21], the radio transceiver consumes $18.8 \mathrm{~mA}(56.4 \mathrm{~mW}$ at $3 \mathrm{~V})$ in the reception mode (including idle listening mode) and $17.4 \mathrm{~mA}(52.2 \mathrm{~mW}$ at $3 \mathrm{~V})$ in the transmission mode (ITX). Note that energy consumption of a sensor node in sleep mode is not zero but less than several tens of $\mu \mathrm{W}$ in real-world WSNs. However, it is negligible and can be set to zero because this research focuses not on calculation of real-world energy consumption of sensor devices but on energy performance comparison of WSN MAC schemes.
Symbols and Variables section shows symbols and variables used in our performance evaluation. Constant time values are derived for the CC2420 radio chip by assuming a total packet length of 10 bytes and 30 300 bytes for a control message and a data packet, respectively, and data rate of $250 \mathrm{kbps}$. The transmission range with these parameter values is about 56 meters. And, considering the size of the target field as well as the transmission rage of a sensor node, we set the number of node groups in the target field to be 10, default buffer size of all the sensor nodes to be $2048 \mathrm{~KB}$, and $k_{2}$ to be 1 for the performance evaluation and analysis of the MAC schemes.

Differently from the existing MACs, GM-MAC sends data only to upstream nodes, which leads $P(\rho)=1$ and $P(\tau)=0$ in Symbols and Variables section. Also, depending on the amount of data in the buffer as well as control signals, GM-MAC takes different actions as follows.

Case 1. This is the case that the node has accumulated data the size of which is less than buffer threshold and does not receive RTS or R_RTS from any downstream node.

Case 2. This is the case that the node has accumulated data the size of which is less than buffer threshold and receives RTS or R_RTS from any downstream node.

Case 3. This is the case that the node has accumulated data the size of which is equal to or greater than buffer threshold and does not receive RTS or R_RTS from any downstream node.

Case 4. This is the case that the node has accumulated data the size of which is equal to or greater than buffer threshold and receives RTS or R_RTS after data transmission.

4.1. Numerical Analysis and Experiments for Active Time of Wireless Sensor Devices. Active time of a sensor device in GM-MAC is categorized into four cases as follows.

(1) Active time $T_{A_{\alpha}}$ for Case 1. Consider

$$
T_{A_{\alpha}}=P(\alpha) \times T_{G}
$$

(2) Active time $T_{A_{\beta}}$ for Case 2. Consider

$$
T_{A_{\beta}}=P(\beta) \times\left(t_{p}+t_{R}+t_{C}+t_{l}+T_{G}\right) .
$$

(3) Active time $T_{A_{\gamma}}$ for Case 3. Consider

$$
T_{A_{\gamma}}=P(\gamma) \times\left(t_{p}+t_{R}+t_{C}+t_{l}+T_{G}\right) .
$$

(4) Active time $T_{A_{\delta}}$ for Case 4. Consider

$$
T_{A_{\delta}}=P(\delta) \times\left[2\left(t_{p}+t_{R}+t_{C}+t_{l}\right)+T_{G}\right] .
$$




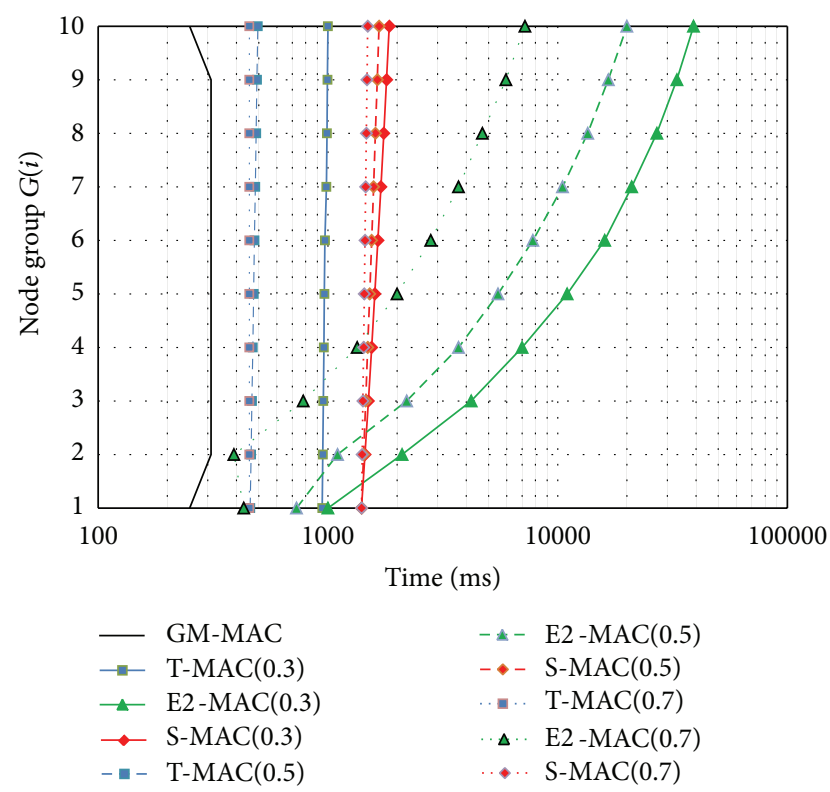

FIgURE 6: Average active time of nodes in node group $G(i)$. Numbers in parentheses denote the data transmission probability $P(\rho)$ from node group $G(i+1)$ to node group $G(i)$.

Therefore, total average active time of the entire sensor devices $T_{\mathrm{GM}_{\text {tot }}}$ in the target field is

$$
\begin{aligned}
& T_{\mathrm{GM}_{\mathrm{tot}}} \\
& =P(\alpha) \sum_{i=1}^{n} i T_{G_{i}}+[P(\beta)+P(\gamma)] \\
& \quad \times \sum_{i=1}^{n} i\left(t_{p_{i}}+t_{R_{i}}+t_{C_{i}}+t_{l_{i}}+T_{G_{i}}\right) \\
& +P(\delta) \sum_{i=1}^{n} i\left[2\left(t_{p_{i}}+t_{R_{i}}+t_{C_{i}}+t_{l_{i}}\right)+T_{G_{i}}\right] \\
& =\frac{n(n+1)}{2}\left\{\left[P(\alpha) T_{G}\right]+[P(\beta)+P(\gamma)]\right. \\
& \quad \times\left(t_{p}+t_{R}+t_{C}+t_{l}+T_{G}\right) \\
& \left.+P(\delta)\left[2\left(t_{p}+t_{R}+t_{C}+t_{l}\right)+T_{G}\right]\right\},
\end{aligned}
$$

where $i$ denotes the node group and $n$ is the total number of node groups. In (7), we assume that all the time variables of sensor devices have the same value, which leads the individual time variables $t_{p_{i}}, t_{R_{i}}, t_{C_{i}}, t_{l_{i}}$, and $T_{G_{i}}$ to be simplified to $t_{p}, t_{R}, t_{C}, t_{l}$, and $T_{G}$, respectively. Based on (7), we can easily derive average active time of each node group. Figure 6 shows average active time of nodes in node group $G(i)$. In addition, in order to evaluate performance features under the situation of randomly distributed sensor nodes and varying traffic conditions, we have applied several data transmission probabilities $P(\rho)$ of $0.3,0.5$, and 0.7 .
For the existing MAC schemes, we can similarly derive total average active time of the entire sensor devices as follows.

Total average active time $T_{S_{\text {tot }}}$ of the entire sensor nodes for S-MAC is

$$
\begin{aligned}
T_{s_{\mathrm{tot}}} & =P(\rho) \sum_{i=1}^{n} i T_{d_{i}}+P(\tau) \sum_{i=1}^{n} i T_{d_{i}}+P(1-\rho-\tau) \sum_{i=1}^{n} i T_{d_{i}} \\
& =\frac{n(n+1)}{2}[P(\rho)+P(\tau)+P(1-\rho-\tau)] T_{d} .
\end{aligned}
$$

Total average active time $T_{T_{\text {tot }}}$ of the entire sensor nodes for $\mathrm{T}-\mathrm{MAC}$ is

$$
\begin{aligned}
T_{T_{\mathrm{tot}}}= & P(\rho) \\
& \cdot\left[P(p) \sum_{i=1}^{n} i T_{0_{i}}+P(q) \sum_{i=1}^{n} i\left(t_{p_{i}}+t_{R_{i}}+t_{c_{i}}+t_{l_{i}}+T_{0_{i}}\right)\right] \\
& +P(\tau)\left[P(p) \sum_{i=1}^{n} i T_{0_{i}}\right. \\
& \left.+P(q) \sum_{i=1}^{n} i\left(t_{p_{i}}+t_{R_{i}}+t_{C_{i}}+t_{l_{i}}+T_{0_{i}}\right)\right] \\
& +P(1-\rho-\tau) \\
& \cdot\left[P(p) \sum_{i=1}^{n} i T_{0_{i}}+P(q) \sum_{i=1}^{n} i\left(t_{p_{i}}+t_{C_{i}}+t_{l_{i}}+T_{0_{i}}\right)\right] \\
= & \frac{n(n+1)}{2}[P(\rho)+P(\tau)+P(1-\rho-\tau)] \\
& \times\left[P(p) T_{0}+P(q)\left(t_{p}+t_{R}+t_{c}+t_{l}+T_{0}\right)\right] .
\end{aligned}
$$

Total average active time $T_{E_{\text {tot }}^{2}}$ of the entire sensor nodes for $\mathrm{E} 2-\mathrm{MAC}$ is

$$
\begin{aligned}
& T_{E_{\mathrm{tot}}^{2}} \\
& \begin{aligned}
=P(\rho) \sum_{i=1}^{n} i\{ & P(\epsilon) T_{E_{i}}+[P(\sigma)+P(\omega)] \\
& \times\left(t_{p_{i}}+t_{R_{i}}+t_{C_{i}}+t_{l_{i}}+T_{E_{i}}\right) \\
& \left.+P(\varphi)\left[2\left(t_{p_{i}}+t_{R_{i}}+t_{C_{i}}+t_{l_{i}}\right)+T_{E_{i}}\right]\right\} \\
+P(\tau) \sum_{i=1}^{n} i\{ & P(\epsilon) T_{E_{i}}+[P(\sigma)+P(\omega)] \\
& \times\left(t_{p_{i}}+t_{R_{i}}+t_{C_{i}}+t_{l_{i}}+T_{E_{i}}\right) \\
& \left.+P(\varphi)\left[2\left(t_{p_{i}}+t_{R_{i}}+t_{C_{i}}+t_{l_{i}}\right)+T_{E_{i}}\right]\right\}
\end{aligned}
\end{aligned}
$$




$$
\begin{aligned}
& +P(1-\rho-\tau) \\
& \cdot \sum_{i=1}^{n} i\left\{P(\epsilon) T_{E_{i}}+[P(\sigma)+P(\omega)]\right. \\
& \times\left(t_{p_{i}}+t_{R_{i}}+t_{C_{i}}+t_{l_{i}}+T_{E_{i}}\right) \\
& \left.+P(\varphi)\left[2\left(t_{p_{i}}+t_{R_{i}}+t_{C_{i}}+t_{l_{i}}\right)+T_{E_{i}}\right]\right\} \\
& =\frac{n(n+1)}{2}[P(1-\rho-\tau)] \\
& \times\left\{P(\epsilon) T_{E}+[P(\sigma)+P(\omega)]\left(t_{p}+t_{R}+t_{C}+t_{l}+T_{E}\right)\right. \\
& \left.+P(\varphi)\left[2\left(t_{p}+t_{R}+t_{C}+t_{l}\right)+T_{E_{i}}\right]\right\} .
\end{aligned}
$$

Figure 6 shows the average active time of nodes in node group $G(i)$. In S-MAC, T-MAC, and E2-MAC, the higher the data transmission probability of $P(\rho)$ increases, the lower the active time of the sensor devices becomes. GM-MAC, however, shows constant active time regardless of $P(\rho)$. This is because GM-MAC always sends the data in the direction of the sink node. From the results, GM-MAC outperforms SMAC, T-MAC, and E2-MAC by about 350\%, 70-300\%, and 40-4700\%, respectively.

4.2. Numerical Analysis and Experiments for Transmission Delays of Wireless Sensor Devices. For the transmission delay evaluation, data are generated with the size ranging from 30 to 300 bytes. Also, we assume that the control signals are 10 bytes in length, and data traffic has the Poisson distribution. Again, data transmission delays of a sensor device are divided into four cases.

(1) Data transmission delay $T_{D_{\mathrm{GM}_{P}(\alpha)}}$ for Case 1. Consider

$$
T_{D_{\mathrm{GM}_{P(\alpha)}}}=P(\alpha) \times T_{G}
$$

(2) Data transmission delay $T_{D_{\mathrm{GM}_{P}(\beta)}}$ for Case 2. Consider

$$
T_{D_{\mathrm{GM} P(\beta)}}=P(\beta) \times\left(t_{p}+t_{R}+t_{C}+t_{l}+t_{\mathrm{ack}}+T_{G}\right) .
$$

(3) Data transmission delay $T_{D_{\mathrm{GM}_{P}(\gamma)}}$ for Case 3. Consider

$$
T_{D_{\mathrm{GM}_{P}(\gamma)}}=P(\gamma) \times\left(t_{p}+t_{R}+t_{C}+t_{l}+t_{\mathrm{ack}}+T_{G}\right) .
$$

(4) Data transmission delay $T_{D_{\mathrm{GM}_{P}(\delta)}}$ for Case 4. Consider

$$
T_{D_{\mathrm{GM}_{P}(\delta)}}=P(\delta) \times\left(t_{p}+2 t_{R}+2 t_{C}+2 t_{l}+2 t_{\mathrm{ack}}+T_{G}\right) .
$$

Therefore, total average hop-by-hop data transmission delay of the entire sensor devices in the target field $T_{D_{\mathrm{GM}}}$ is

$$
\begin{aligned}
& T_{D_{\mathrm{GM}}} \\
& \begin{aligned}
=\sum_{i=1}^{n} i\{[ & \left.P(\alpha) \times T_{G_{i}}\right]+[P(\beta)+P(\gamma)] \\
& \times\left(t_{p_{i}}+t_{C_{i}}+t_{R_{i}}+t_{l_{i}}+t_{\mathrm{ack}_{i}}+T_{G_{i}}\right) \\
& +P(\delta) \times\left(t_{p_{i}}+2 t_{C_{i}}+2 t_{R_{i}}+2 t_{l_{i}}\right. \\
& \left.\left.+2 t_{\mathrm{ack}_{i}}+T_{G_{i}}\right)\right\} .
\end{aligned}
\end{aligned}
$$

Again, based on (12), we can easily derive average hop-by-hop data transmission delay of each node group. For the existing MAC schemes, we can similarly derive total average hop-byhop data transmission delay of the entire sensor devices as follows.

Total average data transmission delay $T_{D_{S}}$ of the entire sensor devices for S-MAC is

$$
\begin{aligned}
T_{D_{S}}= & P(\rho) \sum_{i=1}^{n}\left(t_{p_{i}}+t_{R_{i}}+t_{c_{i}}+t_{l_{i}}+t_{\mathrm{ack}_{i}}\right) \\
& +P(\tau) \sum_{i=1}^{n}\left(t_{p_{i}}+t_{R_{i}}+t_{c_{i}}+t_{l_{i}}+t_{\mathrm{ack}_{i}}\right) \\
& +P(1-\rho-\tau) \sum_{i=1}^{n}\left(t_{p_{i}}+t_{R_{i}}+t_{c_{i}}+t_{l_{i}}+t_{\mathrm{ack}_{i}}\right) \\
= & \frac{n(n+1)}{2}[P(\rho)+P(\tau)+P(1-\rho-\tau)] \\
& \times\left(t_{p}+t_{R}+t_{c}+t_{l}+t_{\mathrm{ack}}\right) .
\end{aligned}
$$

Total average data transmission delay $T_{D_{T}}$ of the entire sensor devices for T-MAC is

$$
\begin{aligned}
& T_{D_{T}} \\
& =P(\rho) \sum_{i=1}^{n}\left[P(p) T_{0_{i}}\right. \\
& \left.+P(q)\left(t_{p_{i}}+t_{R_{i}}+t_{C_{i}}+t_{l_{i}}+t_{\mathrm{ack}_{i}}+T_{0_{i}}\right)\right] \\
& +P(\tau) \sum_{i=1}^{n}\left[P(p) T_{0_{i}}\right. \\
& \left.+P(q)\left(t_{p_{i}}+t_{R_{i}}+t_{C_{i}}+t_{l_{i}}+t_{\mathrm{ack}_{i}}+T_{0_{i}}\right)\right] \\
& +P(1-\rho-\tau) \\
& \cdot \sum_{i=1}^{n}\left[P(p) T_{0_{i}}+P(q)\left(t_{p_{i}}+t_{R_{i}}+t_{C_{i}}+t_{l_{i}}+t_{\mathrm{ack}_{i}}+T_{0_{i}}\right)\right] \\
& =\frac{n(n+1)}{2}[P(\rho)+P(\tau)+P(1-\rho-\tau)] \\
& \times\left[P(p) T_{0}+P(q)\left(t_{p}+t_{R}+t_{C}+t_{l}+t_{\mathrm{ack}}+T_{0}\right)\right] .
\end{aligned}
$$


Total average data transmission delay $T_{D_{E^{2}}}$ of the entire sensor devices for E2-MAC is

$$
\begin{aligned}
& T_{D_{E^{2}}} \\
& =P(\rho) \sum_{i=1}^{n}\left\{\left[P(\epsilon) \times T_{E_{i}}\right]+[P(\omega)+P(\sigma)]\right. \\
& \times\left(t_{p_{i}}+t_{C_{i}}+t_{R_{i}}+t_{l_{i}}+t_{\mathrm{ack}_{i}}+T_{E_{i}}\right)+P(\varphi) \\
& \left.\times\left(t_{p_{i}}+2 t_{C_{i}}+2 t_{R_{i}}+2 t_{l_{i}}+2 t_{\text {ack }_{i}}+T_{E_{i}}\right)\right\} \\
& +P(\tau) \sum_{i=1}^{n}\left\{\left[P(\epsilon) \times T_{E_{i}}\right]+[P(\omega)+P(\sigma)]\right. \\
& \times\left(t_{p_{i}}+t_{C_{i}}+t_{R_{i}}+t_{l_{i}}+t_{\mathrm{ack}_{i}}+T_{E_{i}}\right)+P(\varphi) \\
& \left.\times\left(t_{p_{i}}+2 t_{C_{i}}+2 t_{R_{i}}+2 t_{l_{i}}+2 t_{\text {ack }_{i}}+T_{E_{i}}\right)\right\} \\
& +P(1-\rho-\tau) \\
& \cdot \sum_{i=1}^{n}\left\{\left[P(\epsilon) \times T_{E_{i}}\right]+[P(\omega)+P(\sigma)]\right. \\
& \times\left(t_{p_{i}}+t_{C_{i}}+t_{R_{i}}+t_{l_{i}}+t_{\mathrm{ack}_{i}}+T_{E_{i}}\right)+P(\varphi) \\
& \left.\times\left(t_{p_{i}}+2 t_{C_{i}}+2 t_{R_{i}}+2 t_{l_{i}}+2 t_{\text {ack }_{i}}+T_{E_{i}}\right)\right\} \\
& =\frac{n(n+1)}{2}[P(\rho)+P(\tau)+P(1-\rho-\tau)] \\
& \times\left\{\left[P(\epsilon) \times T_{E_{i}}\right]+[P(\omega)+P(\sigma)]\right. \\
& \times\left(t_{p}+t_{C}+t_{R}+t_{l}+t_{\text {ack }}+T_{E}\right) \\
& \left.+P(\varphi) \times\left(t_{p}+2 t_{C}+2 t_{R}+2 t_{l}+2 t_{\text {ack }}+T_{E}\right)\right\} .
\end{aligned}
$$

Figure 7 shows average data transmission delays of nodes in node group $G(i)$. GM-MAC has very low transmission delays compared to other schemes. Particularly, GM-MAC shows quite narrow variations in data transmission delays. This is because GM-MAC does not depend on the data transmission probability $P(\rho)$, sets buffer threshold value of a sensor device to be reciprocal to the distance from the sink node, and sends the data only in the direction of the sink node. Other schemes show wide variations in delay times depending on the separation distance from the sink node as well as the data transmission probability $P(\rho)$.

\subsection{Numerical Analysis and Experiments for Control Signal} Overheads of Wireless Sensor Devices. Unnecessary control overhead should be avoided as it is one of the main factors that consume device's energy. All the contention-based approaches need at least 2 control signals to transmit or receive data, RTS and CTS. In addition to these control signals, GM-MAC needs 2 more control signals for both R_RTS and C_CTS. It, however, uses less control signals than the other schemes, because it does not send data to

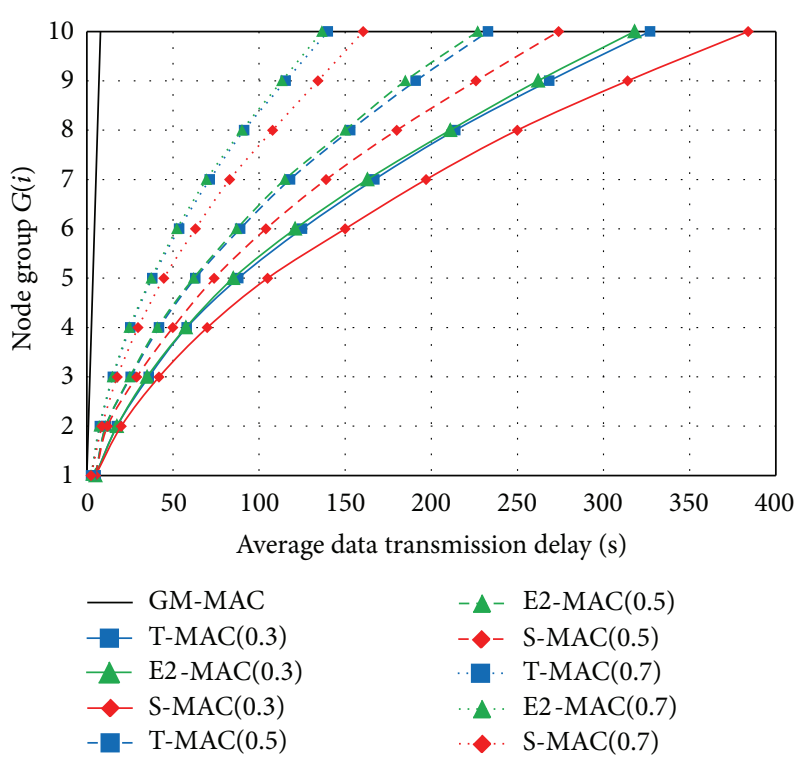

Figure 7: Average data transmission delays of nodes in node group $G(i)$.

the neighboring nodes in the same group and downstream node groups. Total average control signal overhead of the entire sensor devices $O_{\mathrm{CP}_{\mathrm{GM}}}$ in the target field is

$$
\begin{aligned}
O_{\mathrm{CP}_{\mathrm{GM}}} & =\sum_{i=1}^{n} i\left\{[P(\beta)+P(\gamma)] C_{P_{i}}+P(\delta) 2 C_{P_{i}}\right\} \\
& =\sum_{i=1}^{n} i\{[P(\beta)+P(\gamma)] 2 i+P(\delta) 4 i\} \\
& =\left(n^{2}+n\right)\{[P(\beta)+P(\gamma)]+2 P(\delta)\},
\end{aligned}
$$

where $C_{P_{i}}$ is the number of control packets issued by a node in node group $G(i)$ to send the data to the other node. Differently from the existing schemes, GM-MAC sends and receives control packets only when it actually sends or receives data with neighboring nodes. Furthermore, as buffer thresholds are differentially applied to node groups, the total number of control packets decreases in the whole WSN. From (13), we can easily derive average number of control packets of each node group. For the existing MAC schemes, we can similarly derive average control signal overhead of the entire sensor devices as follows.

Total average control signal overhead $O_{\mathrm{CP}_{S}}$ of the entire sensor nodes for S-MAC is

$$
\begin{aligned}
O_{\mathrm{CP}_{S}} & =P(\rho) \sum_{i=1}^{n} C_{P_{i}}+P(\tau) \sum_{i=1}^{n} C_{P_{i}}+P(1-\rho-\tau) \sum_{i=1}^{n} C_{P_{i}} \\
& =P(\rho) \sum_{i=1}^{n} 2 i+P(\tau) \sum_{i=1}^{n} 2 i+P(1-\rho-\tau) \sum_{i=1}^{n} 2 i \\
& =\left(n^{2}+n\right)[P(\rho)+P(\tau)+P(1-\rho-\tau)] .
\end{aligned}
$$


Total average control signal overhead $\mathrm{O}_{\mathrm{CP}_{T}}$ of the entire sensor nodes for T-MAC is

$$
\begin{aligned}
O_{\mathrm{CP}_{T}}= & P(\rho) \sum_{i=1}^{n} P(q) C_{P_{i}}+P(\tau) \sum_{i=1}^{n} P(q) C_{P_{i}}+P(1-\rho-\tau) \\
& \cdot \sum_{i=1}^{n} P(q) C_{P_{i}} \\
= & P(\rho) \sum_{i=1}^{n} P(q) 2 i+P(\tau) \sum_{i=1}^{n} P(q) 2 i+P(1-\rho-\tau) \\
& \cdot \sum_{i=1}^{n} P(q) 2 i \\
= & P(q)\left(n^{2}+n\right)[P(\rho)+P(\tau)+P(1-\rho-\tau)] .
\end{aligned}
$$

Total average control signal overhead $O_{\mathrm{CP}_{E^{2}}}$ of the entire sensor nodes for E2-MAC is

$$
\begin{aligned}
O_{\mathrm{CP}_{E^{2}}}= & P(\rho) \sum_{i=1}^{n}\left\{[P(\omega)+P(\sigma)] C_{P_{i}}+\left[P(\varphi) 2 C_{P_{i}}\right]\right\} \\
& +P(\tau) \sum_{i=1}^{n}\left\{[P(\omega)+P(\sigma)] C_{P_{i}}+\left[P(\varphi) 2 C_{P_{i}}\right]\right\} \\
& +[1-P(\rho)-P(\tau)] \\
& \cdot \sum_{i=1}^{n}\left\{[P(\omega)+P(\sigma)] C_{P_{i}}+\left[P(\varphi) 2 C_{P_{i}}\right]\right\} \\
= & P(\rho) \sum_{i=1}^{n}\{[P(\omega)+P(\sigma)] 2 i+[P(\varphi) 4 i]\} \\
& +P(\tau) \sum_{i=1}^{n}\{[P(\omega)+P(\sigma)] 2 i+[P(\varphi) 4 i]\} \\
& +[1-P(\rho)-P(\tau)] \\
& \cdot \sum_{i=1}^{n}\{[P(\omega)+P(\sigma)] 2 i+[P(\varphi) 4 i]\} \\
= & n(n+1) \times\{P(\rho)+P(\tau)+[1-P(\rho)-P(\tau)]\} \\
& \times\{[P(\omega)+P(\sigma)]+2 P(\varphi)\} .
\end{aligned}
$$

Figure 8 shows average number of control packets of nodes in node group $G(i)$. From the results, GM-MAC outperforms the existing schemes by about $20-1000 \%$. Note that T-MAC needs the heaviest overheads depending on the probability of sending data in the direction of the sink node as well as the data transmission probability $P(\rho)$.

4.4. Numerical Analysis and Experiments for Energy Consumption of Wireless Sensor Devices. Considering the energy consumption of the entire sensor devices in the target

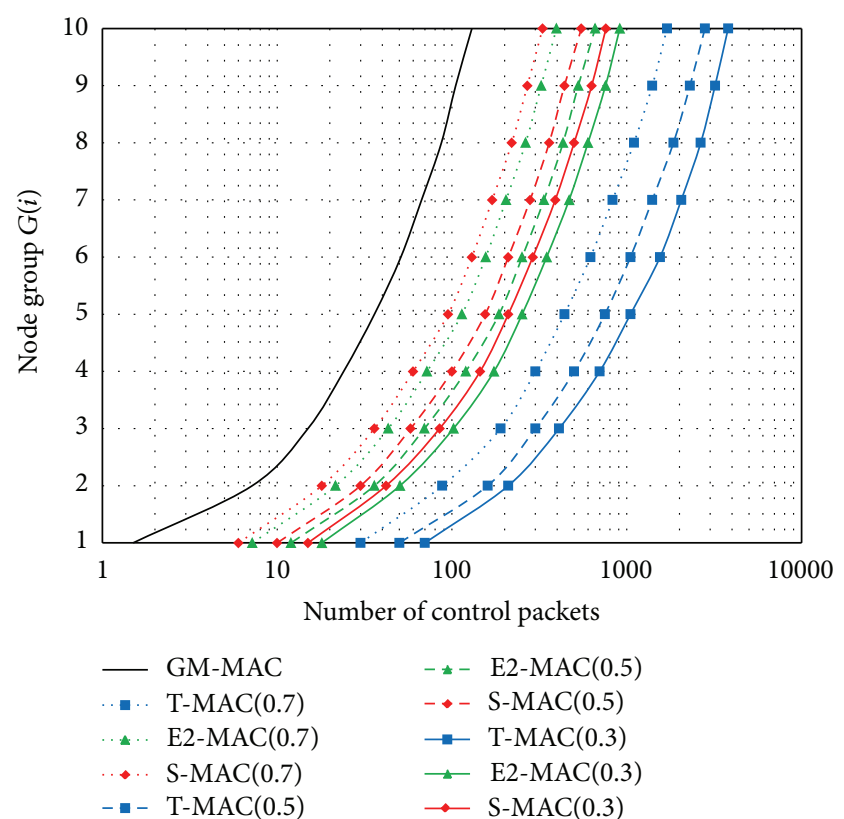

FIGURE 8: Average number of control packets of nodes in node group $G(i)$.

field is most important as it determines the lifetime of the corresponding WSN. Again, the energy consumption of a sensor device in GM-MAC is categorized into four categories.

(1) Energy consumption $E_{\mathrm{GM}_{P(\alpha)}}$ for Case 1. Consider

$$
E_{\mathrm{GM}_{P(\alpha)}}=P(\alpha)\left[\left(t_{p}+T_{G}\right) E_{\mathrm{id}}\right]
$$

(2) Energy consumption $E_{\mathrm{GM}_{P(\beta)}}$ for Case 2. Consider

$$
E_{\mathrm{GM}_{P(\beta)}}=P(\beta)\left[t_{p} E_{\mathrm{id}}+t_{c}\left(E_{r}+E_{s}\right)+t_{r} E_{r}+T_{G} E_{\mathrm{id}}\right] .
$$

(3) Energy consumption $E_{\mathrm{GM}_{P(\gamma)}}$ for Case 3. Consider

$$
E_{\mathrm{GM}_{P(\gamma)}}=P(\gamma)\left[t_{p} E_{\mathrm{id}}+t_{c}\left(E_{r}+E_{s}\right)+t_{s} E_{s}+T_{G} E_{\mathrm{id}}\right] .
$$

(4) Energy consumption $E_{G M_{P(\delta)}}$ for Case 4. Consider

$$
\begin{aligned}
& E_{G M_{P(\delta)}} \\
& \quad=P(\delta)\left[t_{p} E_{\mathrm{id}}+2 t_{c}\left(E_{r}+E_{s}\right)+t_{t} E_{s}+t_{r} E_{r}+T_{G} E_{\mathrm{id}}\right] .
\end{aligned}
$$


Therefore, total average energy consumption $E_{\mathrm{GM}}$ of the entire sensor devices in the target field is

$$
\begin{aligned}
& E_{\mathrm{GM}} \\
& =\sum_{i=1}^{n} i\left\{P(\alpha)\left[\left(t_{p_{i}}+T_{G_{i}}\right) E_{\mathrm{id}}\right]\right. \\
& +P(\beta)\left[t_{p_{i}} E_{\mathrm{id}}+t_{c_{i}}\left(E_{r}+E_{s}\right)+t_{r_{i}} E_{r}+T_{G_{i}} E_{\mathrm{id}}\right] \\
& +P(\gamma)\left[t_{p_{i}} E_{\mathrm{id}}+t_{c_{i}}\left(E_{r}+E_{s}\right)+t_{t_{i}} E_{s}+T_{G_{i}} E_{\mathrm{id}}\right] \\
& +P(\delta)\left[t_{p_{i}} E_{\mathrm{id}}+2 t_{c_{i}}\left(E_{r}+E_{s}\right)+t_{t_{i}} E_{s}\right. \\
& \left.\left.+t_{r_{i}} E_{r}+T_{G_{i}} E_{\mathrm{id}}\right]\right\} \text {. }
\end{aligned}
$$

Note that the energy consumption evaluations have been done by considering only the power consumption of radio chipset. That is, the results are not for the whole sensor device itself. Considering most energy of a sensor node (more than $93 \%$ of its battery power) is consumed when it operates its radio module, it does not lack legitimacy.

From (18), we can easily derive average energy consumption of each node group. For the existing MAC schemes, we can similarly derive total average energy consumption of the entire sensor nodes as follows.

Total average energy consumption $E_{S}$ of the entire sensor nodes for S-MAC is

$$
\begin{gathered}
E_{s}=P(\rho) \sum_{i=1}^{n} i\left\{t_{p_{i}} E_{\mathrm{id}}+t_{c_{i}}\left(E_{s}+E_{r}\right)+t_{t_{i}} E_{s}+t_{r_{i}} E_{r}\right. \\
\left.+t_{\mathrm{ack}_{i}} E_{s}+E_{\mathrm{id}}\left[T_{A}-\left(2 t_{c_{i}}+t_{t_{i}}+t_{r_{i}}\right)\right]\right\} \\
+P(\tau) \sum_{i=1}^{n} i\left\{t_{p_{i}} E_{\mathrm{id}}+t_{c_{i}}\left(E_{s}+E_{r}\right)+t_{t_{i}} E_{s}+t_{r_{i}} E_{r}\right. \\
\left.+t_{\mathrm{ack}_{i}} E_{s}+E_{\mathrm{id}}\left[T_{A}-\left(2 t_{c_{i}}+t_{t_{i}}+t_{r_{i}}\right)\right]\right\} \\
+P(1-\rho-\tau) \\
+\sum_{i=1}^{n} i\left\{t_{p_{i}} E_{\mathrm{id}}+t_{c_{i}}\left(E_{s}+E_{r}\right)+t_{t_{i}} E_{s}+t_{r_{i}} E_{r}\right. \\
\left.+t_{\mathrm{ack}_{i}} E_{s}+E_{\mathrm{id}}\left[T_{A}-\left(2 t_{c_{i}}+t_{t_{i}}+t_{r_{i}}\right)\right]\right\} .
\end{gathered}
$$

Total average energy consumption $E_{T}$ of the entire sensor nodes for T-MAC is

$$
\begin{aligned}
& E_{T} \\
& =P(\rho) \\
& \quad \cdot \sum_{i=1}^{n} i\left\{P(p)\left[\left(t_{p_{i}}+T_{\mathrm{O}_{i}}\right) E_{\mathrm{id}}\right]+P(q)\right. \\
& \left.\quad \cdot\left[t_{p_{i}} E_{\mathrm{id}}+t_{c_{i}}\left(E_{r}+E_{s}\right)+t_{t_{i}} E_{s}+t_{\mathrm{ack}_{i}} E_{r}+T_{\mathrm{O}_{i}} E_{\mathrm{id}}\right]\right\}
\end{aligned}
$$

$$
\begin{aligned}
& +P(\tau) \\
& \cdot \sum_{i=1}^{n} i\left\{P(p)\left[\left(t_{p_{i}}+T_{\mathrm{O}_{i}}\right) E_{\mathrm{id}}\right]+P(q)\right. \\
& \left.\quad \cdot\left[t_{p_{i}} E_{\mathrm{id}}+t_{c_{i}}\left(E_{r}+E_{s}\right)+t_{t_{i}} E_{s}+t_{\mathrm{ack}_{i}} E_{r}+T_{\mathrm{O}_{i}} E_{\mathrm{id}}\right]\right\} \\
& +P(1-\rho-\tau) \\
& \cdot \sum_{i=1}^{n} i\left\{P(p)\left[\left(t_{p_{i}}+T_{\mathrm{O}_{i}}\right) E_{\mathrm{id}}\right]+P(q)\right. \\
& \left.\quad \cdot\left[t_{p_{i}} E_{\mathrm{id}}+t_{c_{i}}\left(E_{r}+E_{s}\right)+t_{t_{i}} E_{s}+t_{\mathrm{ack}_{i}} E_{r}+T_{\mathrm{O}_{i}} E_{\mathrm{id}}\right]\right\}
\end{aligned}
$$

Total average energy consumption $E_{E^{2}}$ of the entire sensor nodes for E2-MAC is

$$
E_{E^{2}}=P(\rho) \sum_{i=1}^{n} i \widetilde{\lambda}+P(\tau) \sum_{i=1}^{n} i \widetilde{\lambda}+P(1-\rho-\tau) \sum_{i=1}^{n} i \widetilde{\lambda},
$$

where

$$
\begin{aligned}
\tilde{\lambda}=\{ & P(\epsilon)\left[T_{E} E_{\mathrm{id}}\right]+P(\sigma) \\
& \cdot\left[t_{p} E_{\mathrm{id}}+t_{c}\left(E_{s}+E_{r}\right)+t_{r} E_{r}+t_{\mathrm{ack}} E_{s}+T_{E} E_{\mathrm{id}}\right] \\
& +P(\varphi)\left[t_{p} E_{\mathrm{id}}+t_{c}\left(E_{s}+E_{r}\right)+t_{t} E_{s}+t_{\mathrm{ack}} E_{r}+T_{E} E_{\mathrm{id}}\right] \\
& +P(\omega)\left[t_{p} E_{\mathrm{id}}+2 t_{c}\left(E_{s}+E_{r}\right)+t_{t} E_{s}+t_{r} E_{r}\right. \\
& \left.\left.+t_{\mathrm{ack}}\left(E_{r}+E_{s}\right)+T_{E} E_{\mathrm{id}}\right]\right\} .
\end{aligned}
$$

At this point, we have to note which sensor nodes are the most critical devices for the lifetime of the whole WSN. Obviously, sensor nodes located nearer to the sink node consume much more energy than those located farther from the sink node in S-MAC, T-MAC, and E2-MAC schemes. This is because sensor nodes located nearer to the sink node collect and receive more data from the downstream nodes and then deliver them to their upstream nodes compared to those located farther from the sink node, which is the intrinsic feature of wireless sensor networks. As a result, sensor nodes in node group $G(1)$ are the first to run short of their energy in the target field. On the other hand, in the proposed GM-MAC scheme, sensor nodes located farthest from the sink node are the first to run short of their energy, because they have the smallest buffer threshold values in the target field. That is, sensor nodes far from the sink node have more chances to send their data to the upstream nodes as GM-MAC sets buffer threshold value of a sensor device to be reciprocal to the distance from the sink node as previously mentioned.

Figure 9 shows average energy consumption of nodes in node group $G(1)$ for S-MAC, T-MAC, and E2-MAC and node group $G(10)$ for GM-MAC when CBR or Poisson data traffics are generated by sensor devices. More concretely, we have compared average energy consumption of the most critical nodes in node group $G(1)$ for the existing MAC schemes 


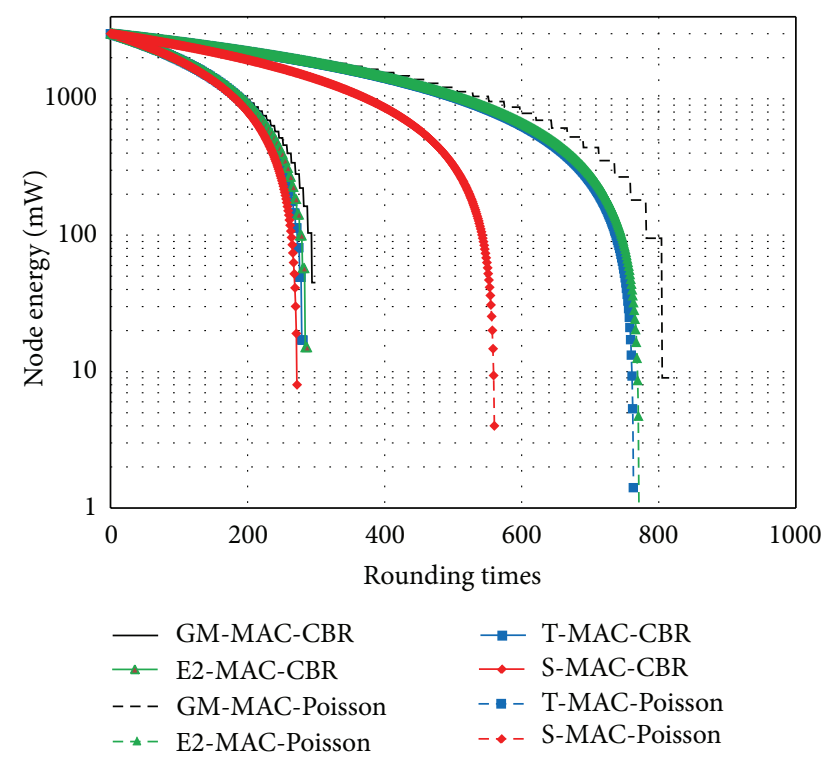

Figure 9: Average energy consumption of S-MAC, T-MAC, and E2-MAC for sensor nodes in node group $G(1)$ and average energy consumption of GM-MAC for sensor nodes in node group $G(10)$.

with average energy consumption of the least critical nodes in node group $G(10)$ for the proposed GM-MAC scheme. If there is no intermediary node to the sink node in WSN, there is no way to collect and receive any interesting data in the target field. From these results, it has been verified that GMMAC outperforms S-MAC, T-MAC, and E2-MAC in energy efficiency by about $10-50 \%, 10 \%$, and $5-10 \%$, on average, respectively. S-MAC survives until about 270 rounding times with CBR traffics and about 540 rounding times with Poisson traffics. The term "rounding time" is defined to be the minimum number of participation of any sensor device to send or receive data. T-MAC survives until about 280 and 780 rounding times with $\mathrm{CBR}$ and Poisson traffics, respectively. T-MAC outperforms S-MAC as it modifies the duty cycle to decrease unnecessary idle listening. E2-MAC shows similar performance compared to T-MAC with CBR traffics, but it is preferable to T-MAC for data traffics with random distribution like Poisson. Finally, GM-MAC outlasts both T$\mathrm{MAC}$ and E2-MAC by more than 20 rounding times.

Note that GM-MAC is specifically designed not only to save the energy of individual nodes but also to maintain the distribution of node energy as evenly as possible in the whole WSN. Obviously, the rounding time of S-MAC, T-MAC, and E2-MAC in Figure 9 means the lifetime of the corresponding WSN. Even if many other sensor devices have enough energy to send and receive data, they have no way to communicate with the sink node. In GM-MAC, however, sensor nodes farthest away from the sink node (nodes in node group $G(10)$ in this case) exhaust their energy first. This means that all the other sensor nodes in node groups $G(1)$ through $G(9)$ can still do their role in the target field. We have proven that nodes in node group $G(9)$ exhaust their energy in the next time, then nodes in node group $G(8), G(7)$, and so on. These results are omitted for the page limitation. As a result, we can conclude that GM-MAC is considerably robust and can maximize the lifetime of the whole WSN.

\section{Conclusion}

This paper presents GM-MAC, a novel, sustainable, and selfconfigurable contention-based MAC that establishes node groups by using preamble signal to effectively manage data transmission, hence the limited energy resources of wireless sensor devices. Sensor devices using GM-MAC experience minimal node activations, transmission delays, and control overheads as data traffic is optimally delivered in the direction of the sink node. Moreover, by employing variable length buffer threshold values, sensor devices nearer to the sink node preserve their energy in such a way to maximize the lifetime of the whole WSN systems. In addition, by employing improved control signals, sensor nodes could efficiently communicate with their neighboring nodes. Lastly, GM-MAC copes with topology changes by using preamble, node group, and buffer threshold recalculation procedure. This feature is especially important for the WSN systems which have mobile sensor devices. Experimental results on four main indices have verified that the proposed GM-MAC is definitely superior to the existing contention-based MACs. Therefore, GM-MAC is expected to be widely used for various types of wireless sensor communication devices. Advanced MAC protocol design issues like routing and database management policy in WSN are to be addressed in a further study.

\section{Symbols and Variables}

$\begin{array}{ll}t_{p}: & \text { Contention time }(0.384 \mathrm{~ms}) \\ t_{R}: & \text { RTS Tx/Rx time }(0.128 \mathrm{~ms}) \\ t_{C}: & \text { CTS Tx/Rx time }(0.128 \mathrm{~ms}) \\ t_{l}: & \text { Data Tx/Rx time }(0.512 \mathrm{~ms}) \\ T_{G}: & \text { Timeout time for GM-MAC }(243.75 \mathrm{~ms}) \\ t_{c}: & \text { Control signal Tx/Rx time }(0.128 \mathrm{~ms}) \\ t_{r}: & \text { Data Rx time }(0.512 \mathrm{~ms}) \\ t_{t}: & \text { Data Tx time }(0.512 \mathrm{~ms}) \\ t_{\text {ack }}: & \text { Acknowledgment Tx/Rx time }(0.128 \mathrm{~ms}) \\ T_{A}: & \text { Active time of duty cycle } \\ T_{d}: & T_{\text {radio off }}-T_{\text {radio on }} \text { during the duty cycle } \\ T_{O}: & \text { Timeout time for T-MAC }(243.75 \mathrm{~ms}) \\ T_{E}: & \text { Timeout time for E2-MAC }(243.75 \mathrm{~ms}) \\ T_{\mathrm{id}}: & \text { Idle listening time in active mode of duty } \\ & \text { cycle } \\ E_{r}: & \text { Power consumption during data reception } \\ E_{s}: & \text { Power consumption during data } \\ E_{\mathrm{id}} & \text { transmission } \\ P(\rho): & \text { Power consumption during idle listening } \\ P(\tau): & \text { Data transmission probability from } \\ P(1-\rho-\tau): & \text { Data transmission probability from } G(i) \text { to } \\ P(\alpha): & \text { Data transmission probability in the same } \\ & \text { Probability of }\left(B_{\text {data }}<B_{G(i)}\right) \text { and (receiving } \\ & \text { no RTS or R_RTS) for GM-MAC } \\ & \end{array}$


$P(\beta)$ : Probability of $\left(B_{\text {data }}<B_{G(i)}\right)$ and (receiving RTS or R_RTS) for GM-MAC

$P(\gamma)$ : Probability of $\left(B_{\text {data }} \geq B_{G(i)}\right)$ and (receiving no RTS or R_RTS) for GM-MAC

$P(\delta)$ : Probability of $\left(B_{\text {data }} \geq B_{G(i)}\right)$ and (receiving RTS or R_RTS) for GM-MAC

$P(p)$ : Probability of no data ready for transmission for T-MAC

$P(q)$ : Probability of any data ready for transmission for T-MAC

$P(\epsilon)$ : Probability of $\left(B_{\text {data }}<B_{\text {threshold }}\right)$ and (receiving no RTS) for E2-MAC

$P(\omega)$ : Probability of $\left(B_{\text {data }}<B_{\text {threshold }}\right)$ and (receiving RTS) for E2-MAC

$P(\sigma)$ : Probability of $\left(B_{\text {data }} \geq B_{\text {threshold }}\right)$ and (receiving no RTS) for E2-MAC

$P(\varphi)$ : of $\left(B_{\text {data }} \geq B_{\text {threshold }}\right)$ and (receiving RTS) for E2-MAC.

\section{Conflict of Interests}

The authors declare that there is no conflict of interests regarding the publication of this paper.

\section{Acknowledgments}

This research was supported by the MSIP (Ministry of Science, ICT and Future Planning), Korea, under the ITRC (Information Technology Research Center) support program (NIPA-2014-H0301-14-1002) supervised by the NIPA (National IT Industry Promotion Agency). Also, this research was supported by Basic Science Research Program through the National Research Foundation of Korea (NRF) funded by the Ministry of Education (2014R1A1A2060035).

\section{References}

[1] K. Sohrabi, J. Gao, V. Ailawadhi, and G. J. Pottie, "Protocols for self-organization of a wireless sensor network," IEEE Personal Communications, vol. 7, no. 5, pp. 16-27, 2000.

[2] IEEE Std. 802.11-2012, Wireless LAN Medium Access Control (MAC) and Physical Layer (PHY) Specifications, 2012.

[3] H. Zhang, J. Llorca, C. C. Davis, and S. D. Milner, "A selforganization framework for wireless Ad Hoc networks as small worlds," IEEE Transactions on Mobile Computing, vol. 11, no. 7, pp. 1207-1222, 2012.

[4] J.-C. Liu and C.-D. Chung, "Distributed estimation in a wireless sensor network using hybrid MAC," IEEE Transactions on Vehicular Technology, vol. 60, no. 7, pp. 3424-3435, 2011.

[5] X. Yang and N. Vaidya, "On physical carrier sensing in wireless ad hoc networks," in Proceedings of the IEEE INFOCOM, pp. 174-185, March 2005.

[6] H. Su and X. Zhang, "Battery-dynamics driven tdma mac protocols for wireless body-area monitoring networks in healthcare applications," IEEE Journal on Selected Areas in Communications, vol. 27, no. 4, pp. 424-434, 2009.

[7] J. Du and W. Shi, "App-MAC: an application-aware eventoriented MAC protocol for multimodality wireless sensor networks," IEEE Transactions on Vehicular Technology, vol. 57, no. 6, pp. 3723-3731, 2008.
[8] F. Iannello, O. Simeone, and U. Spagnolini, "Medium access control protocols for wireless sensor networks with energy harvesting," IEEE Transactions on Communications, vol. 60, no. 5, pp. 1381-1389, 2012.

[9] G. Lu, B. Krishnamachari, and C. S. Raghavendra, "An adaptive energy efficient and low latency MAC for data gathering in wireless sensor networks," in Proceedings of the IEEE 18th International Parallel and Distributed Processing Symposium (IPDPS '04), pp. 22-30, April 2004.

[10] S. S. Kulkarni, "TDMA services for sensor networks," in Proceedings of the IEEE 24th International Conference on Distributed Computing Systems Workshops (ICDCS 04), pp. 139172, March 2004.

[11] K.-W. Chin, "Pairwise: a time hopping medium access control protocol for wireless sensor networks," IEEE Transactions on Consumer Electronics, vol. 55, no. 4, pp. 1898-1906, 2009.

[12] A. El-Hoiydi, "Aloha with preamble sampling for sporadic traffic in ad hoc wireless sensor networks," in Proceedings of the IEEE International Conference on Communications (ICC '02), vol. 5, pp. 3418-3423, 2002.

[13] A. El-Hoiydi, J.-D. Decotignie, C. Enz, and E. Le Roux, "Poster abstract: WiseMAC, an ultra low power MAC protocol for the wiseNET wireless sensor network," in Proceedings of the 1st International Conference on Embedded Networked Sensor Systems (SenSys '03), pp. 302-303, November 2003.

[14] W. Ye, J. Heidemann, and D. Estrin, "An energy-efficient MAC protocol for wireless sensor networks," in Proceedings of the 21st Annual Joint Conference of the IEEE Computer and Communications Societies (INFOCOM '02), vol. 3, pp. 15671576, IEEE, June 2002.

[15] T. van Dam and K. Langendoen, "An adaptive energy-efficient MAC protocol for wireless sensor networks," in Proceedings of the 1st International Conference on Embedded Networked Sensor Systems (SenSys '03), pp. 171-180, Association for Computing Machinery, November 2003.

[16] J.-H. Kim, H.-N. Kim, S.-G. Kim, S.-J. Choi, and J.-Y. Lee, "Advanced MAC protocol with energy-efficiency for wireless sensor networks," in Proceedings of the International Conference on Information Networking (ICOIN '05), pp. 283-292, Jeju Island, Republic of Korea, February 2005.

[17] W. Ye, J. Heidemann, and D. Estrin, "Medium access control with coordinated adaptive sleeping for wireless sensor networks," IEEE/ACM Transactions on Networking, vol. 12, no. 3, pp. 493-506, 2004.

[18] X. Yang and N. H.vaidya, "A wakeup scheme for sensor networks: achieving balance between energy saving and endto-end delay," in Proceedings of the 10th IEEE Real-Time and Embedded Technology and Applications Symposium (RTAS '04), pp. 19-26, May 2004.

[19] M. Dhanaraj, B. S. Manoj, and C. S. R. Murthy, "A new energy efficient protocol for minimizing multi-hop latency in wireless sensor networks," in Proceedings of the 3rd IEEE International Conference on Pervasive Computing and Communications (PerCom '05), pp. 117-126, 2005.

[20] T. Baykas, J. Wang, R. Funada, and A. Rahman, "Preamble design for millimeter-wave single carrier WPANs," in Proceedings of the Vehicular Technology Conference, pp. 1-5, April 2009.

[21] CC2420 data sheet, Chipcon, Texas Instruments. 


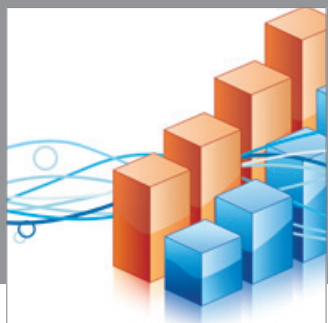

Advances in

Operations Research

mansans

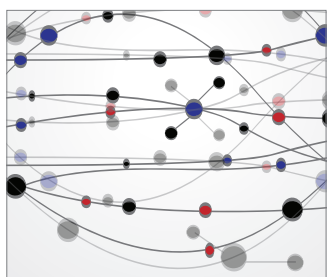

The Scientific World Journal
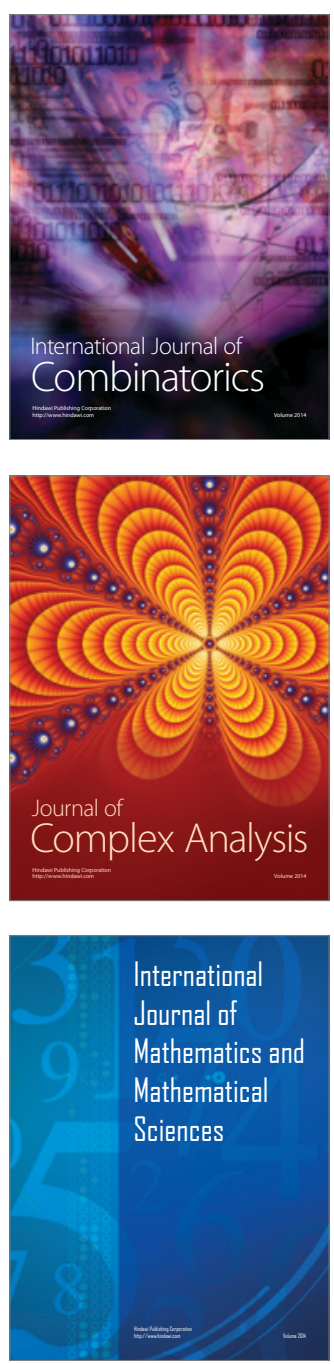
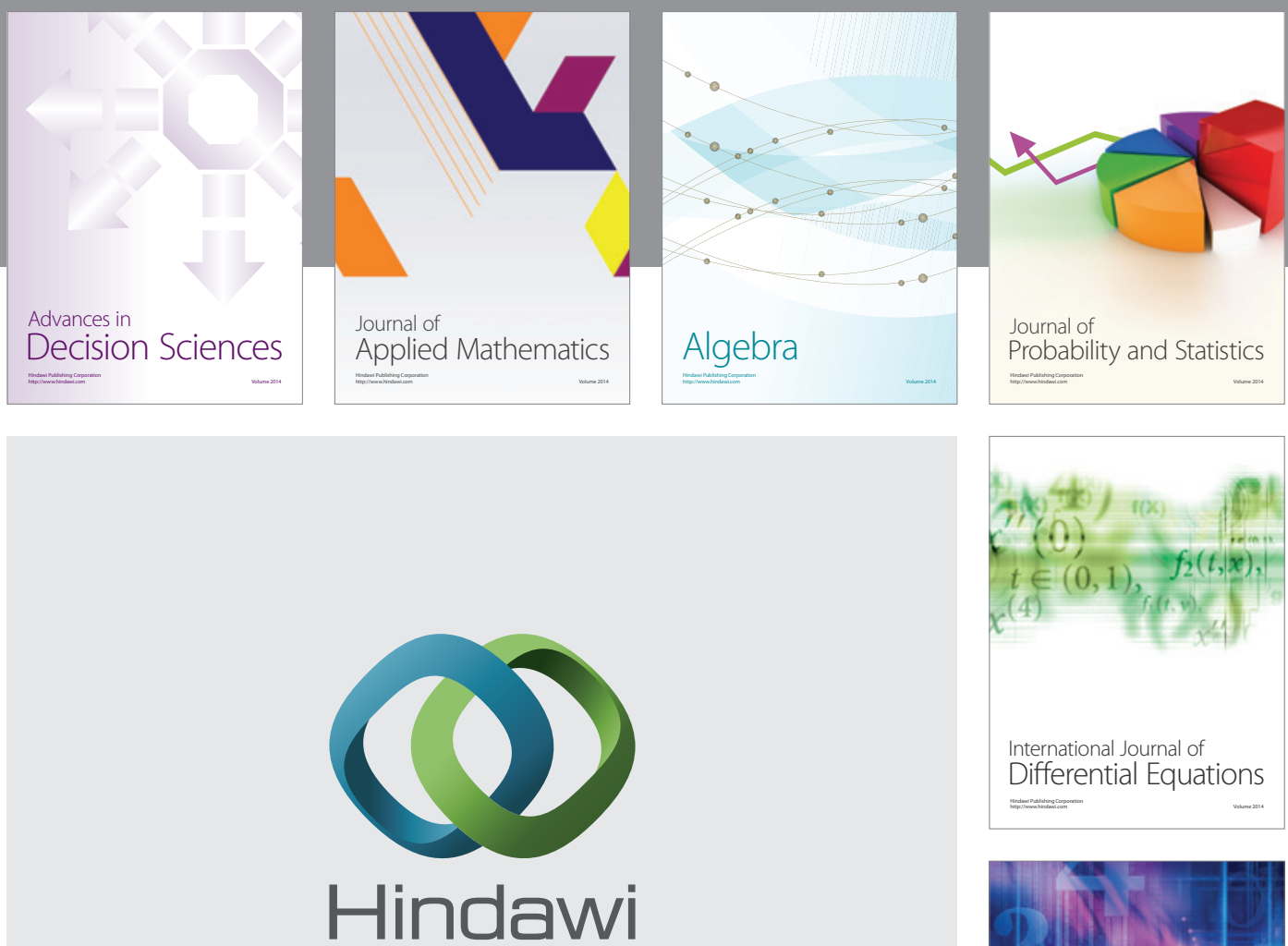

Submit your manuscripts at http://www.hindawi.com
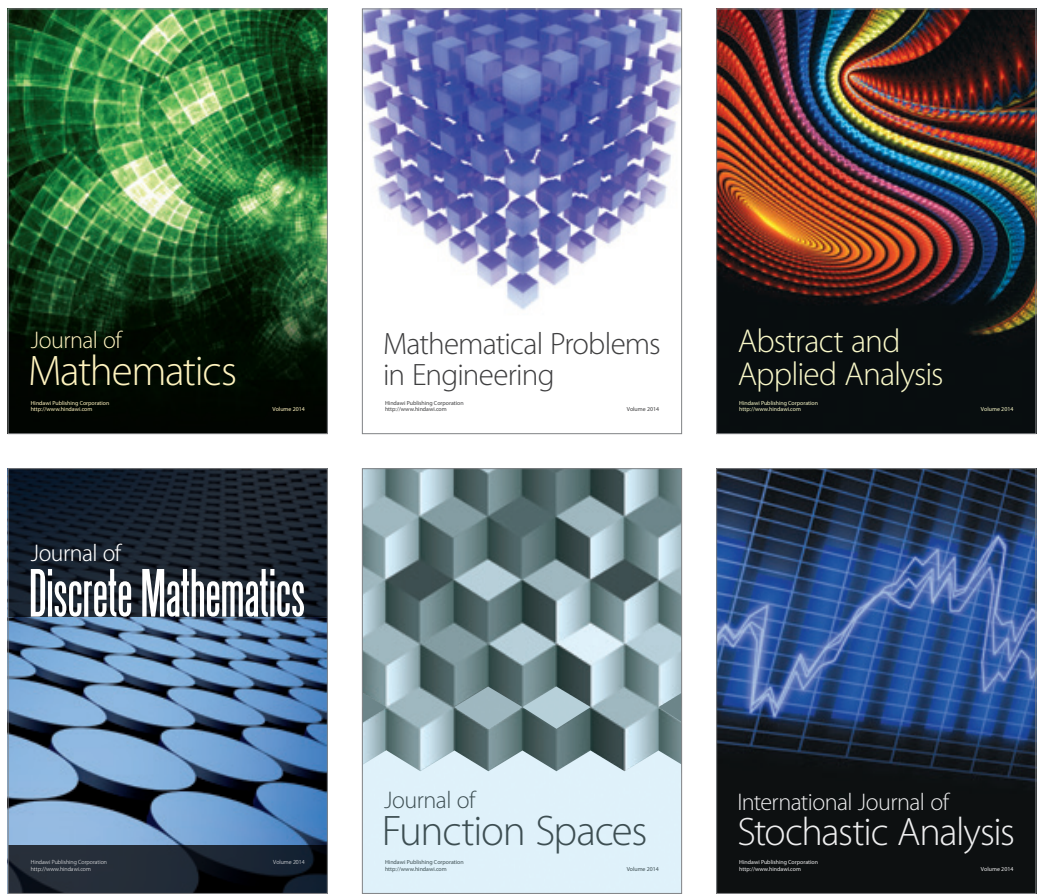

Journal of

Function Spaces

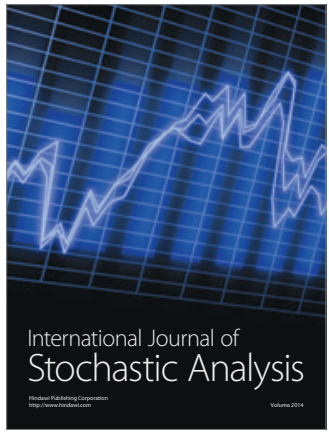

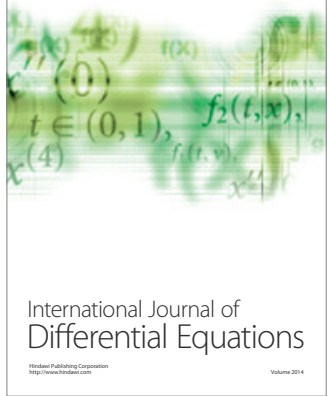
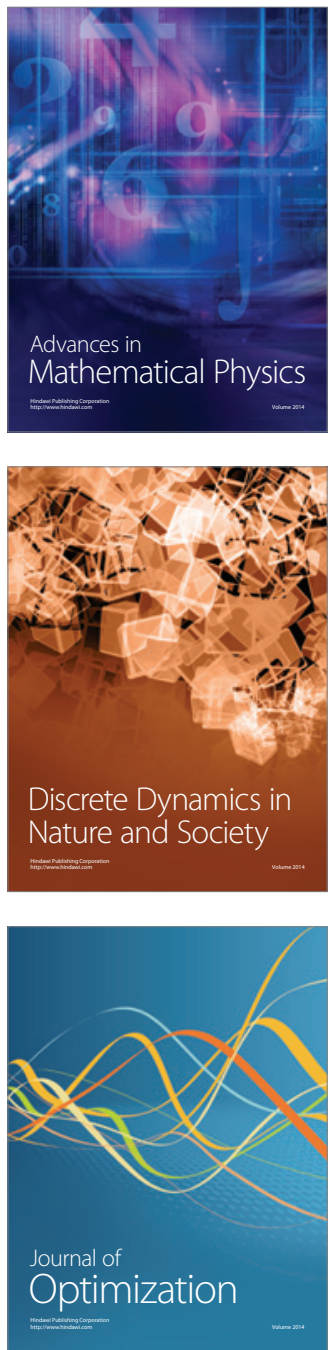